\title{
Applications of Matrix Optics to Acceptance Studies in Low-beta Ion Linacs
}

\author{
K. Joh and J. A. Nolen \\ Argonne National Laboratory \\ Argonne, IL 60439, U. S. A.
}

\section{Abstract}

Four-gap superconducting resonators have been developed at Argonne for use in the low-beta positive ion injector (PII) for ATLAS [1]. 'These structures have been used successfully for ion velocities as low as $0.007 \mathrm{c}$ with $\mathrm{q} / \mathrm{m}=0.1$. First order matrix optics [2] and linear theory for the phase space transformations in accelerating systems [2], [3] are applied to the PII linac which includes low-beta heavy ion If resonators and magnetic solenoids.

These provide a new method to match initial phase space ellipses when used with higher order transfer maps or raytracing calculations. And also we present a quantitative measure of nonlinearities using the concept of rms emittances and deviations of phase space coordinates between linear and nonlinear transformations. As a byproduct a way of identifying the dominant source of nonlinearity of system is indicated.

\section{ACCEPtances}

The maximum emittance of a beam that a system can accept is called the acceptance of the system; the particles within this acceptance will be transmitted without striking the wall. But only some part of the acceptance would transform linearly if there are aberrations.

\section{A. Geometrical Acceptance}

The geometrical acceptance of a system is defined as the acceptance calculated from linear phase space transformation. This is larger than the useful acceptance if nonlinearities are present.

The beam envelope at a position in a system with acceleration is given for an axially symmetric beam in $n$ dimensional phase space by [2]:

$$
x_{2}=\sqrt{(\operatorname{det} M)^{2 / n} \beta_{2} \epsilon_{1}}
$$

where $\operatorname{det} M$ is the determinant of the transfer matrix. And $\beta_{2}, \epsilon_{1}$ are the beta-function at the position 2 and the initial beam emittance in one projected plane, respectively.
The beta-function at point 2 is found if the initial Twiss parameters of the phase space and the transfer map are known. Equation (1) is still valid for a nonaxially symmetric beam if $n=2$.

We define:

$$
\beta^{*}=(\operatorname{det} M)^{2 / n} \beta,
$$

so that $\beta^{*}$ is the effective beta-function.

Then the geometrical transverse acceptance of a system is limited by the maximum $\beta^{*}$ and apertures of optical elements:

$$
\text { GTacc }=\frac{\mathrm{R}^{2}}{\beta_{\max }^{*}} .
$$

where $R$ is the aperture of the optical element at which the effective beta-function has maximum value $\beta_{\max }^{*}$.

For longitudinal phase space there is no physical aperture which limits acceptances, but to maintain the phase focusing aspect of the resonators phase excursions or timeof-flight deviations must be limited. For example if the of phase offset is $\phi_{n}$ for the reference particle, then the deviation of if phase angle should not exceed $\phi_{0}$ for any particle because at zero if phase nonlinearity would be very important. Hence an estimate of the longitudinal acceptance is obtained by requiring:

$$
\Delta t \leq \frac{\phi_{1}}{\omega} \equiv \mathrm{R}_{\text {long }} .
$$

where $\Delta t$ is the deviation of the time-of-flight and $\omega$ is the angular if frequency of a resonator.

Now $R_{\text {long }}$ is analogous to the radial aperture for the transverse case, so we have for an estimate of the longitudinal acceptance:

$$
\text { GLace }=\frac{R_{\text {long }}^{2}}{\beta_{\max }} .
$$

where $R_{\text {long }}$ was defined above and $\beta_{\max }$ is the maximum value of the longitudinal beta-function.

In practice, we find that the longitudinal acceptance defined in this way is a good starting point for determining the linear acceptance as described below.

The geometrical acceptances can be found numerically by fitting the Twiss parameters of the phase spaces with an appropriate optimizer. The transverse or longitudinal 
acceptance is calculated for an assumed initial set of $T$ wiss parameters. Then the optimizer varies the Twiss parameters until the maximum values of the geometrical acceptances are determined.

\section{B. Linear Acceptance}

The nonlinearity of a system was not considered in the geometrical acceptance calculations described above. In practice only a fraction of the geometrical acceptance transforms linearly. To establish a quantitative measure of the degree of nonlinearity, a new quantity, called the deviation of the particle $i$, is defined:

$$
\text { dev. } \equiv \frac{\left|x_{i}^{i}-x_{n}^{i}\right|}{x_{1 m}}
$$

where subscript $l, n$ indicate linear and nonlinear transformations of the particle $i, r \in s p e c t i v e l y$, and $x_{1 m}$ means the maximum value of the coordinate $x$ in the linear transformations of the geometrical acceptance.

We introduce a method to find linear acceptances by comparing linear and nonlinear transformations. As an example, let us find the transverse linear acceptance with dev. $\leq 1 \%$ :

- Find the geometrical acceptance using an optimizer.

- Find maximum values of position $x_{m}$ and divergence $x_{m}^{\prime}$ from the linear transformation of the initial geometrical acceptance ellipse.

- Find devistions of phase space coordinates of individual rays with coordinates chosen on a grid bounded by the geometrical phase space ellipse from linear and nonlinear transformations.

- Discard all initial rays for which one of the deviations is greater than $1 \%$ to get the linear acceptance from the remaining rays.

Note that this deviation method gives the area and the orientation of a phase space ellipse. The degree of nonlinearity is controlled through the choice of the magnitude of dev, parameter.

\section{NONLINEARITy OF A System}

For nonlinear systems the increase of rms emittance ("emittance growth") is often used as a quantitative measure of the nonlinearity. Here we examine the degree of correlation between the magnitude of the dev. parameter used above and the amount of emittance growth exhibited by the system.

The rms emittance is defined for a pair of phase space coordinates $x$ and $x^{\prime}$ by [4]:

$$
\epsilon_{r m a}=4 \sqrt{\left\langle x^{2}\right\rangle\left\langle x^{\prime 2}\right\rangle-\left\langle x x^{r}\right\rangle^{2}}
$$

For linear systems the rms emittance is transformed in general [5]:

$$
\epsilon_{r m \cdot 2}=(\operatorname{det} M) \cdot \epsilon_{r m+1} .
$$

where det $M$ is the determinant of a transfer map of a system.

Thus (det $M)^{-1} \epsilon_{r m n}$ is constant of motinn. And we use it to modify the usual definition of normalized emittance:

$$
\epsilon_{n r m s}=(\operatorname{det} M)^{-1} \cdot \epsilon_{r m s} .
$$

Equation (9) would yield the common definition of the normalized emittance for the transverse case if $\epsilon_{\mathrm{rma}}$ were multiplied by the initial value of $\beta_{\gamma}$. Because of the invariance of the normalized emittance under linear transformations it might be used as a measure of the nonlinearity of a system by comparing its values between linear and nonlinear transformations.

We define differences of normalized emittances between linear and nonlinear transformations:

$$
\delta \epsilon^{l, l} \equiv \epsilon_{n \mathrm{rma}}^{t, l}-\epsilon_{\mathrm{nrman}}^{\ell, l} .
$$

where superscripts $l, t$ indicate longitudinal and transverse phase spaces, respectively, and 0 indicates initial emittance.

Below we explore the correlation between the normalized rms emittance accepted by the system and the magnitude of the two maximum values of the dev. parameters. We also examine the relations between the rms emittance growth and the magnitude of the dev. parameters permitted in the transverse and longitudinal dimensions, including the effects of cross terms.

\section{ApPlication}

We applied these concepts to the first section of the PII linac, which consists of three magnetic solenoids (s) and three low-beta if resonators (I) as shown in Figure 1. The strengths of magnetic solenoids are adjusted to give beam waist conditions at the center of each resonator. Because of the low velocities the transfer maps are calculated analytically point-by-point through the system.

As an example a ${ }^{2: 14} U^{2 \cdot 1+}$ beam with an incident velocity $0.0085 \mathrm{c}$ was used. The rf settings of field gradients and initial if phase angles for the three resonators are:

I1: $4.5 \mathrm{MV} / \mathrm{m}\left(-10^{\circ}\right)$, I2-1: $3.0\left(-15^{\circ}\right)$, I2-2: $3.0\left(-15^{\circ}\right)$.

The calculated geometrical transverse acceptance of the system is $263 \pi \mathrm{mm} \cdot \mathrm{mr}$ which is shown as the large ellipse in the Figure 2. The individual rays inside this ellipse are transformed nonlinearly with up to 3rd order off-axial fields of the if resonators and magnetic solenoids to find linear acceptances for different deviations defined in the equation (6). In these raytracing calculations the initial longitudinal phase space area was assumed to be zero so that nonlinear coupling from transverse to longitudinal dimensions is emphasized.

Figure 2 shows the portion of the geometrical acceptance which transforms linearly with the dev. parameter limited to less than 0.01 ; the corresponding areas accepted are $263 \pi \mathrm{mm} \cdot \mathrm{mr}$ (geometrical) and $70 \pi \mathrm{mm} \cdot \mathrm{mr}$ (linear). A 
calculation of the accepted rms emittance as a function of the maximum value of the dev. parameter over the range from dev. $=0.01$ to 0.10 shows a nearly linear relationship with the value of the linear rms acceptance increasing to $220 \pi \mathrm{mm} \cdot \mathrm{mr}$ at dev. $=0.10$. Figure 3 shows the details for dev. $\leq \mathbf{0 . 0 5}$. Similarly, we evaluated the rms emittance growth of the transverse and longitudinal coordinates as a function of the dev. parameters in the transverse case. It was found, in agreement with previous experience with ATLAS calculations, that for very small nonlinearity in the transverse map, there are much more significant nonlinearities appearing in the longitudinal dimension due to second-order cross terms. (The lowest order aberrations in transverse focusing are third order.) This is seen in Figure 2 where the distortion in the transverse ellipse is very small $\left(\delta \epsilon^{\ell}=0.05 \pi \mathrm{mm} \cdot \mathrm{mr}\right)$ whereas the increase in longitudinal emittance is significant $\left(\delta \epsilon^{\prime}=40 \pi \mathrm{keV} \cdot \mathrm{nsec}\right)$.

One interesting thing is the noticeable difference of the orientations of the geometrical and linear phase space ellipses shown in Figure 2. To understand this the effective beta-functions for the two different orientations were plotted in the Figure 1. Qualitatively, these curves indicate that nonlinearities are minimized by keeping the effective beta-function small in 11 while allowing it to be larger in s1.

\section{Conclusions}

The method to find linear acceptances and to scale nonlinearites of a system by deviations of linear and nonlinear transformations seems to be well defined. Since this method is applicable to any accelerating or beam transport system it will provide a very easy and fast way to find linear acceptances and sources of nonlinearities, if higher order transfer maps are available.

This work was supported in part by the U.S. Department of Energy, Nucl. Phys. Div, under contract No. W-31-109ENG-38

\section{REFERENCES}

[1] L.M. Bollinger et al. Nuclear Physics, A553, (1993) $859 c-862 c$, and references therein.

[2] K. Joh, Michigan State University. Ph.D. Dissertation, (1993).

[3] D.R. Douglas, J. Kewisch, and R.C. York. Proceedings of the 1988 Linear Accelerator Conference, Williamsberg, Virginia, (1988) 328.

[4] P.M. Lapostolle. IEEE Trans. Nucl. Sci., NS-18, No. 3, (1971) 1101.

[5] Alex J. Dragt, Filippo Neri, Govindan Rangarajan. Physical Review A, Vol.45, Number 4 (1991) 2572.

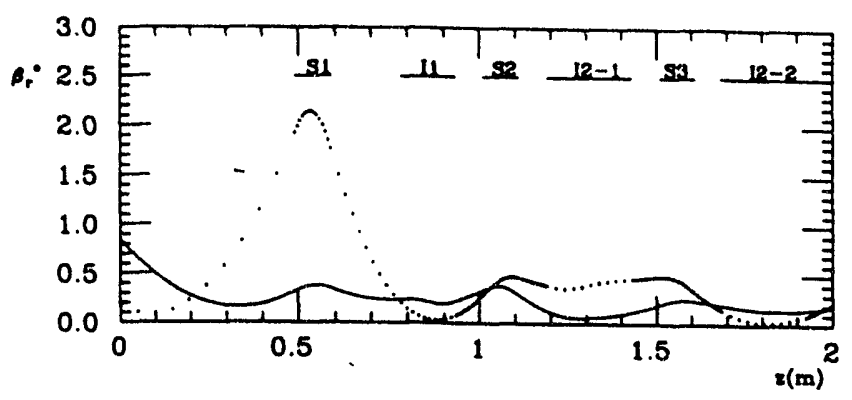

Figure 1: The system configurations and the effective betafunctions for $T$ wiss parameters from the geometrical acceptance (solid) and the linear acceptance (dotted).
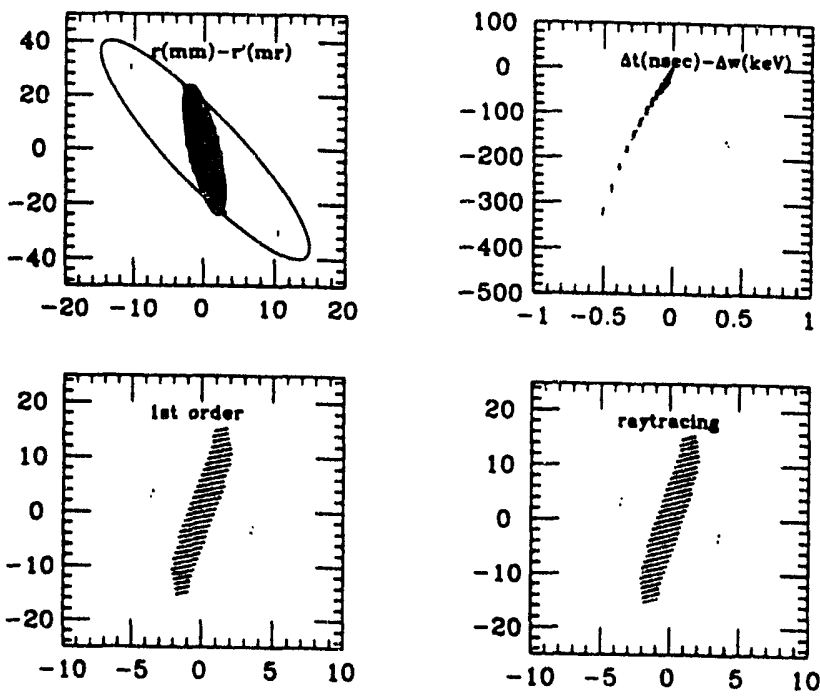

Figure 2: The phase space transformations of linear acceptance by linear (1st order) and nonlinear(raytracing) transformations with dev. $=1 \%$. The emittance induced in longitudinal phase space via nonlinear coupling with transverse coordinates is shown in the top right plot.
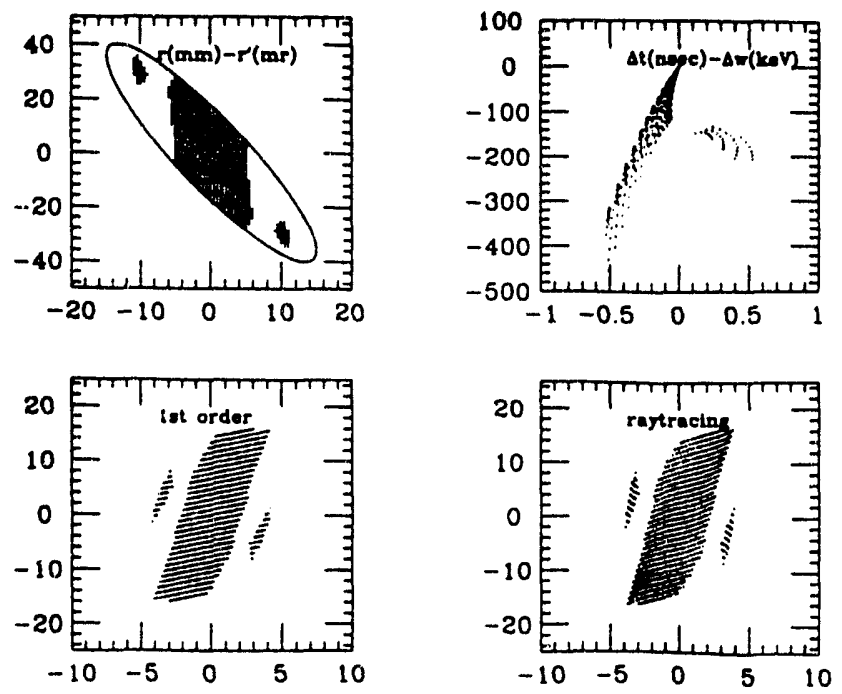

Figure 3: The phase space transformations of linear acceptance by linear (1st order) and nonlinear(raytracing) transformations with dev. $=5 \%$. 

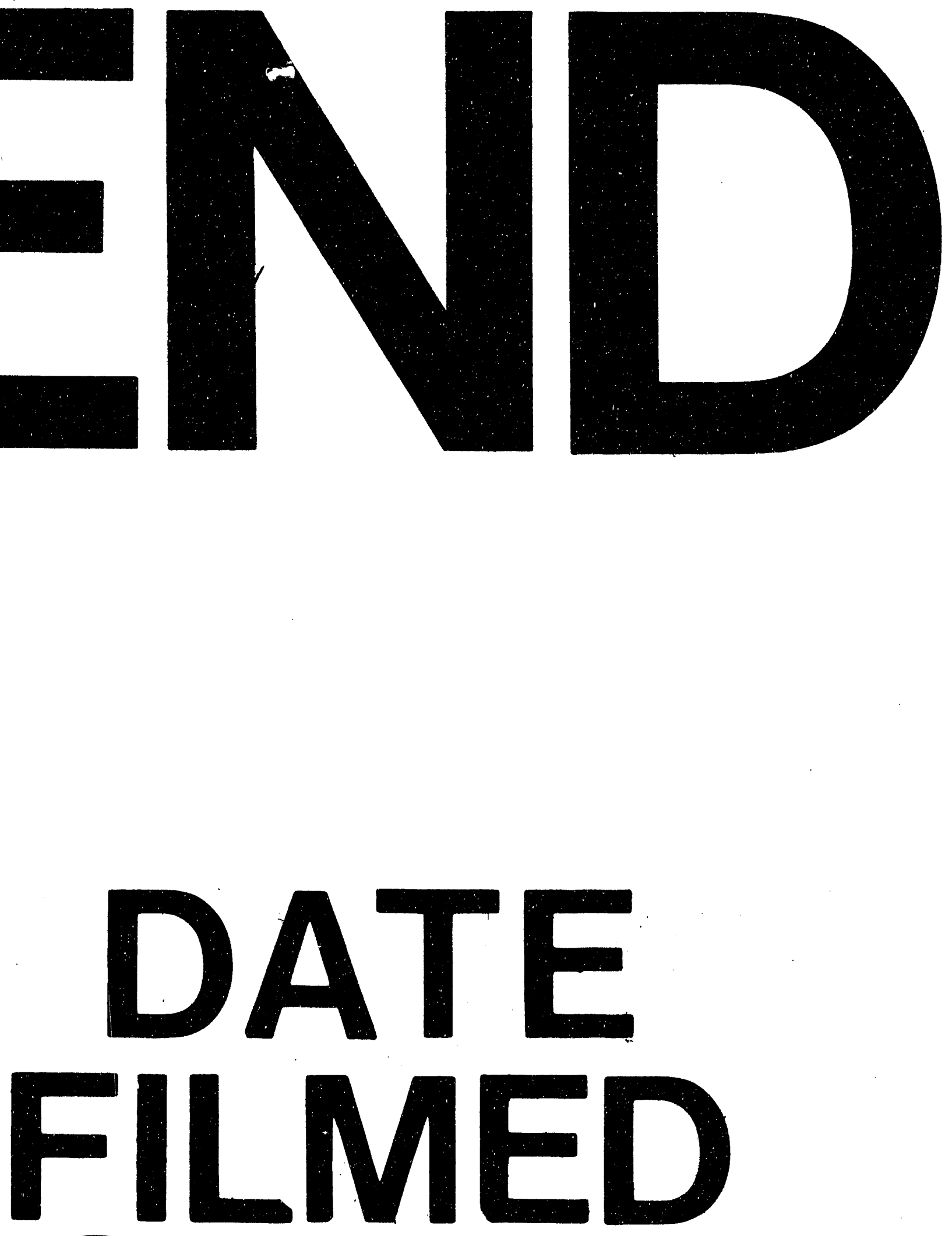

1

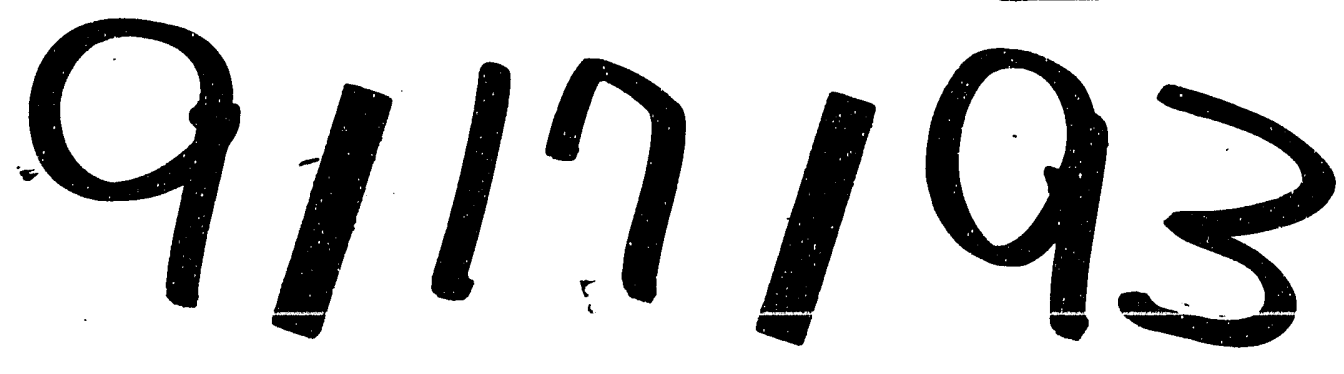


